\title{
Estresse mental em atletas de elite: Uma revisão sistemática
}

\author{
Mental stress in elite athletes: A systematic review \\ Estrés mental en atletas de élite: Una revisión sistemática
}

Recebido: 05/06/2021 | Revisado: 16/06/2021 | Aceito: 17/06/2021 | Publicado: 02/07/2021

\author{
Emanuelly Rocha Farias \\ ORCID: https://orcid.org/0000-0002-9723-7375 \\ Centro Universitário Santo Agostinho, Brasil \\ E-mail: farias.manu102@gmail.com \\ Mauro Fernando Lima da Silva \\ ORCID: https://orcid.org/0000-0001-8866-1855 \\ Centro Universitário Santo Agostinho, Brasil \\ E-mail: mauropetman@yahoo.com.br
}

\begin{abstract}
Resumo
O estresse mental representa um problema de saúde pública e prática de exercício podem impulsionar melhora a saúde mental. Entretanto, atletas não estão imunes aos desafios de saúde mental, inclusive a alta demanda física de atletas de elite pode influenciar negativamente a saúde mental e ocasionar estresse. Essa relação de estresse mental e atletas de elite ainda não bem compreendida na leitura e recebe pouca atenção de pesquisadores. Desse modo, foi realizado um estudo de revisão sistemática com objetivo de analisar a existência e impactos do estresse mental em atletas de elite. Nessa revisão foram utilizados os seguintes descritores: Stress, Psychological; Athletes; Mental Health; Mental Health Associations. As bases de dados utilizadas foram PubMed, Web of Science e Scopus no idioma inglês com artigos publicados nos últimos cinco anos. Após seleção e síntese qualitativa, sete estudos foram incluídos. Foi constatado que o estresse mental e comum em atletas inclusive em comparação a não atletas. Nos estudos incluídos houve predomínio de jovens mulheres em esportes variados, principalmente esportes de verão como voleibol e atletismo. $\mathrm{O}$ estresse mental parece estar relacionado com carga de treinamento. Estudos com amostras maiores são necessários, em diferentes esportes para verificar com maior rigor metodológico o estresse mental em atletas de elite.
\end{abstract}

Palavras-chave: Estresse psicológico; Atletas; Saúde mental; Associações de saúde mental.

\begin{abstract}
Mental stress represents a public health problem and exercise can boost mental health improvement. However, athletes are not immune to mental health challenges, including the high physical demand of elite athletes can negatively influence mental health and cause stress. This relationship of mental stress and elite athletes is still not well understood in reading and receives little attention from researchers. Thus, a systematic review study was carried out with the objective of analyzing the existence and impacts of mental stress in elite athletes. In this review, the following descriptors were used: Stress, Psychological; Athletes; Mental Health; Mental Health Associations. The databases used were PubMed, Web of Science and Scopus in English with articles published in the last five years. After qualitative selection and synthesis, seven studies were included. It was found that mental stress is common in athletes even compared to non-athletes. In the included studies there was a predominance of young women in varied sports, mainly summer sports such as volleyball and athletics. Mental stress seems to be related to training load. Studies with larger samples are needed in different sports to check mental stress in elite athletes with greater methodological rigor.
\end{abstract}

Keywords: Psychological stress; Athletes; Mental health; Mental health associations.

\section{Resumen}

El estrés mental representa un problema de salud pública y el ejercicio puede impulsar la mejora de la salud mental. Sin embargo, los atletas no son inmunes a los desafíos de salud mental, incluida la alta demanda física de los atletas de élite que puede influir negativamente en la salud mental y causar estrés. Esta relación entre el estrés mental y los atletas de élite aún no se comprende bien en la lectura y recibe poca atención por parte de los investigadores. Así, se realizó un estudio de revisión sistemática con el objetivo de analizar la existencia e impactos del estrés mental en deportistas de élite. En esta revisión se utilizaron los siguientes descriptores: estrés, psicológico; Atletas; Salud mental; Asociaciones de salud mental. Las bases de datos utilizadas fueron PubMed, Web of Science y Scopus en inglés con artículos publicados en los últimos cinco años. Después de la selección y síntesis cualitativa, se incluyeron siete estudios. Se encontró que el estrés mental es común en los atletas incluso en comparación con los no atletas. En los estudios incluidos hubo un predominio de mujeres jóvenes en deportes variados, principalmente deportes de verano como voleibol y atletismo. El estrés mental parece estar relacionado con la carga de entrenamiento. Se necesitan estudios con muestras 
más amplias en diferentes deportes para comprobar el estrés mental en deportistas de élite con mayor rigor metodológico.

Palabras clave: Estrés psicológico; Atletas; Salud mental; Asociaciones de salud mental.

\section{Introdução}

Atletas em esportes de alto rendimento enfrentam uma combinação única de desafios, esgotamento físico, lesões, síndrome do sobretreinamento são alguns fatores de risco para alguns distúrbios de saúde (Åkesdotter et al., 2020; Reardon et al., 2019). Comum em muitos atletas de elite os problemas de saúde mental são definidos como transtornos psiquiátricos e sintomas de sofrimento psíquico com impacto substancial na qualidade de vida, causando prejuízo funcional no trabalho, atividades sociais e outras áreas importantes da vida por duas semanas ou mais (Åkesdotter et al., 2020; Foskett; Longstaff, 2018; Schinke et al., 2018).

A prevalência de problemas na saúde mental pode atingir 50\% dos atletas em algum momento de sua carreira e o pico de início ocorre por volta dos 19 anos (Åkesdotter et al., 2020). É importante ressaltar que entre os jovens os problemas de saúde mental são um dos maiores contribuintes para a carga global de doenças, com aumento exponencial nos últimos anos (Vella et al., 2021).

Pesquisas recentes apontaram vários estressores e desafios únicos que os atletas de alto desempenho enfrentam e que podem explicar parcialmente os problemas de saúde mental, como depressão, ansiedade e estresse (Gorczynski; Coyle; Gibson, 2017). Esses desafios e estressores únicos incluem demandas de competição e treinamento, bem como lidar com lesões e recuperação (Gorczynski; Coyle; Gibson, 2017; Foskett; Longstaff, 2018).

Além disso, alguns atletas de alto rendimento também se preocupam com a imagem corporal, principalmente aqueles que competem em esportes estéticos, como mergulho e ginástica, que podem resultar em transtornos alimentares e uso de substâncias (Gorczynski; Coyle; Gibson, 2017). Existe também preocupações com a aposentadoria, especialmente quando provocadas repentina e inesperadamente, deixam os atletas de alto desempenho sentindo-se vulneráveis e com sintomas depressivos (Gorczynski; Coyle; Gibson, 2017; Vannuccini et al., 2020).

Sintomas mentais, como estresse, podem causar mudanças de atenção, distração e aumento da autoconsciência de que tudo pode interferir no desempenho e predispor um atleta a lesão e má recuperação (Putukian, 2016). Apesar dos riscos e da carga, atualmente, não existe uma estrutura ou modelo de atendimento abrangente para apoiar e responder às necessidades de saúde mental de atletas de elite (Russell et al., 2019), aliado a isso os atletas mantêm e agem de acordo com suas próprias atitudes, crenças e opiniões em relação à saúde mental (Moreland; Coxe; Yang, 2018).

Existem vários motivos pelos quais os atletas de alto desempenho podem desejar não divulgar seu estado de saúde mental e buscar apoio. Assim como na população em geral, eles temem lidar com o estigma associado à doença mental e as consequências que daí decorrem, talvez o mais pertinente seja o fato de os atletas não desejarem interromper suas carreiras atléticas (Gorczynski; Coyle; Gibson, 2017; Van Slingerland et al., 2019).

Mesmo com recente interesse na pesquisa de saúde mental, há uma escassez de dados empíricos robustos sobre os sintomas de estresse mental em atletas competidores atualmente (Purcell et al., 2020), existe deficiência do acompanhamento ideal dos atletas uma forte limitação nas informações sobre saúde mental de uma forma geral (Lebrun; Collins, 2017). Nessa perspectiva, foi realizada uma pesquisa com o objetivo de sistematizar através de estudos publicados em periódicos, a incidência, sintomas e impactos do estresse mental em atletas de alto rendimento.

\section{Metodologia}

Foi realizado uma revisão sistemática de literatura com tema central de analisar o estresse mental em atletas de alto rendimento. O estudo foi elaborado, conforme processo metodológico de Donato e Donato (2019) e de modelo de revisão 
sistemática Cochrane (Ahn; Kang, 2018; Delgado-Rodríguez; Sillero-Arenas, 2018; Zhu, 2020), com os seguintes passos: 1. Formular uma questão de investigação; 2. Realizar um protocolo de investigação, 3. Definir os critérios de inclusão e de exclusão; 4. Desenvolver uma estratégia de pesquisa e pesquisar a literatura; 5. Selecionar os estudos; 6. Avaliação da qualidade dos estudos; 7. Extração dos dados; 8. Síntese dos dados e 9. Disseminação dos resultados.

A pergunta de investigação do processo revisional foi construída através da estratégia PICO (acrônimo para patient, intervention, comparison, outcomes), é descrita na tabela 1.

Para a coleta de dados foram utilizados os descritores oriundos do sistema MeSH (Medical Subject Headings): Stress, Psychological; Athletes; Mental Health; Mental Health Associations. Visando maior direcionamento dos artigos para a questão norteadora da presente revisão será utilizado o operador booleano "AND" e "OR".

Tabela 1. Estratégia PICO para formulação da questão de investigação.

\begin{tabular}{|c|c|c|c|}
\hline $\begin{array}{c}\text { 1. POPULAÇÃO } \\
\text { (PATIENT/PROBLEM) }\end{array}$ & $\begin{array}{c}\text { 2. INTERVENÇÃO } \\
\text { (INTERVENTION) }\end{array}$ & $\begin{array}{c}\text { 3. CONTROLE } \\
\text { (COMPARISION) }\end{array}$ & 4. DESFECHO (OUTCOME) \\
\hline atletas de elite & estresse mental & Sem comparação & associação/relação \\
\hline \multicolumn{2}{|c|}{ PERGUNTA: "Qual a associação/relação do estresse mental em atletas de elite"? }
\end{tabular}

Fonte: Autoria própria.

Os bancos de dados foram acessados de março a maio de 2021, onde foi realizada uma pesquisa nas seguintes bases de dados: PubMed, Web of Science e Scopus. Essas plataformas de dados foram acessadas com auxílio do portal de periódicos da Capes, por meio da Comunidade Acadêmica Federada (CAFe)

Os critérios de inclusão foram artigos publicados na língua inglesa, dos últimos cinco anos, essa data foi escolhida por uma revisão anterior (Rice et al., 2016) incluir estudos até a data de 2016, essa revisão abordou saúde mental de uma forma geral, contudo, desfechos de estresse mental foram abordados. Foram incluídos os desportistas de elite competindo profissionalmente e olímpicos (Stillman et al., 2019). Atletas em nível colegial/universitário não foram incluídos. Foram excluídas produções repetidas (entre as bases de dados); editoriais; avaliações; comentários; artigos não revisados por pares; artigos com foco na população em geral.

Nas bases Web of Science e Scopus a ferramenta de busca avançada foi utilizada para combinar as seguintes entradas com o operador boleano "AND”: (“Athletes”) e (“Stress, Psychological”"; "Mental Health”; "Mental Health Associations”) em todos os campos. Na PubMed, a ferramenta de busca avançada também foi utilizada para conduzir a mesma estratégia de busca utilizada para no campo "tópicos".

Os estudos foram primariamente cruzados entre base de dados para retirada de duplicatas, em seguida foi visto os artigos por títulos e resumo. Posteriormente os artigos remanescentes foram analisados por texto completo, para então ser realizado a síntese qualitativa (com verificação da população, método utilizadas e coleta de dados). A qualidade do estudo foi avaliada quanto ao tipo de estudo e rigor metodológico aplicado, devido à heterogeneidade do desenho do estudo e características das amostras no qual ensaios clínicos randomizados (principal critério de inclusão como rigor metodológico), não foi possível combinar os resultados em uma meta-análise. Consequentemente, os resultados foram descritos relatando dados por meio de uma revisão sistemática. 


\section{Resultados e Discussão}

Com a busca efetuada encontrou-se 7741 artigos, após a aplicação de filtros restaram 1218 artigos, destes 826 no PubMed, 138 no Web of Science e 254 na Scopus. Primeiramente foram analisados os títulos e resumos dos trabalhos, buscando menções dos termos utilizados durante a busca foram removidos 1070 artigos que estava em duas ou mais bases de dados, ou não atendia os critérios de busca com essa remoção permaneceram 50. Em um segundo momento fez-se uma análise dos artigos por meio da leitura e a avaliação da pertinência para essa revisão sistemática, através da leitura dos textos por completo, sendo excluídos 40 artigos, restando, por não responderem à questão de investigação ou por não atenderem a todos os critérios de inclusão, restando 11 estudos para síntese qualitativa, após leitura da metodologia e verificação novamente da população estudada, mais 4 estudos foram excluídos na síntese qualitativa. Com isso, apenas os trabalhos que tinham relação com os termos utilizados na busca e com o objetivo do presente estudo foram utilizados, totalizando 7 artigos nessa pesquisa (Fluxograma 1).

Figura 1. Método de busca utilizado.

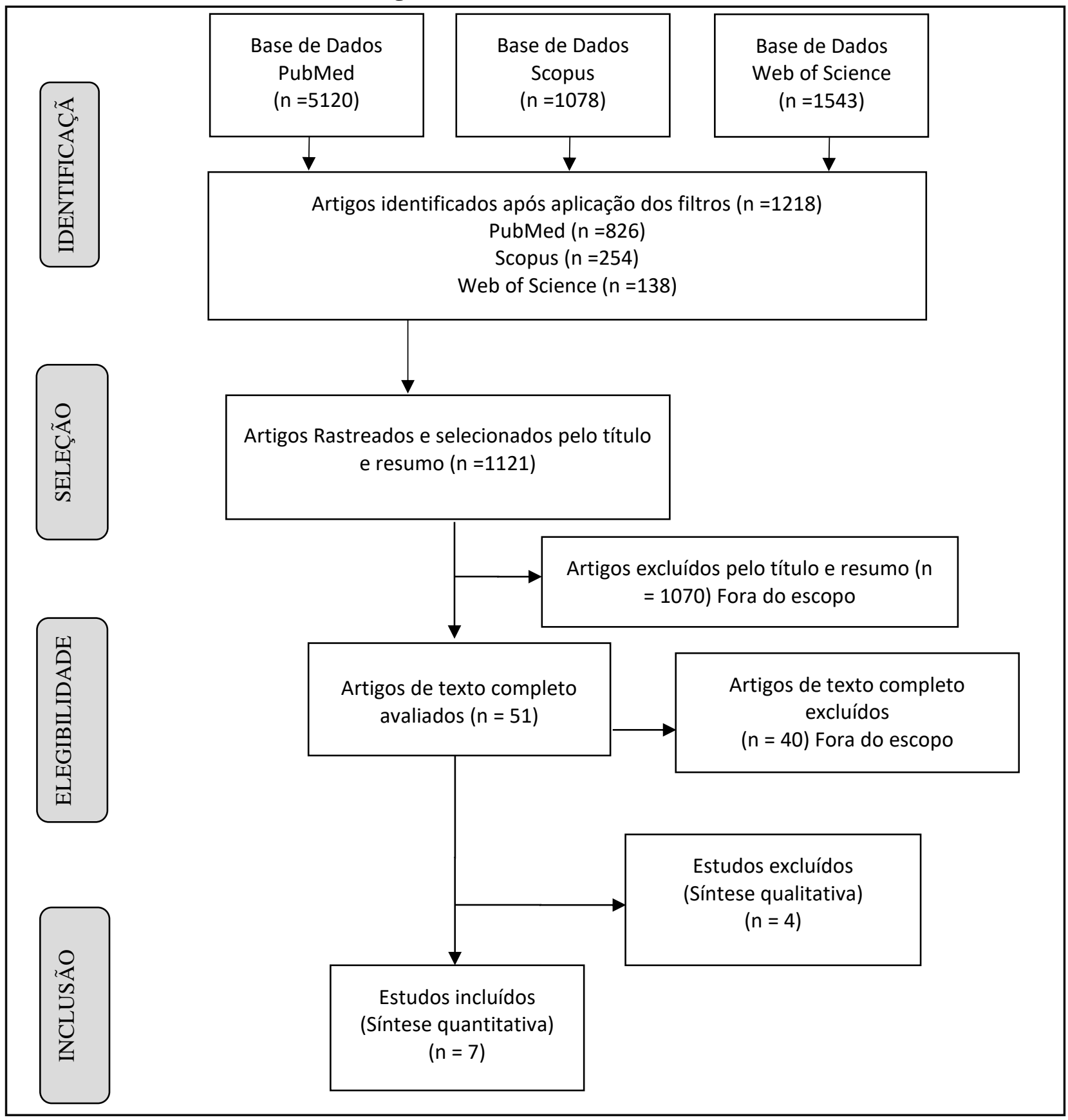


Fonte: Autoria própria baseado no modelo prisma de revisão sistemática adaptação de MOHER et al., (2009).

Os 7 artigos incluídos através da estratégia de busca foram descritos inicialmente na Tabela 2 quanto a fonte, data, objetivo, método, resultados. Houve predomínio de mulheres e grande parte dos estudos tinham mulheres o sexo feminino como amostra total (100\%). Foram encontrados principalmente estudos transversais, e as amostras pesquisadas variaram de 08 a 154 , com idade média variando entre 18 e 25 anos. Os esportes praticados pelos atletas investigados variaram bastante, foi visto esportes individuais como atletismo e coletivos como voleibol, houve predominância também em esporte de verão, somente um estudo mostrou esporte de inverno.

Houve relação entre aumento do estresse e, atletas de elite, verificado inclusive em estudos controlados com a população de não atletas (Dziembowska et al., 2019; Vannuccini et al., 2020). Houve grande variação do método de medição do estresse mental, a medição ocorreu em dados subjetivos como em escalas (Belz et al., 2018; Codonhato et al., 2018; Dziembowska et al., 2019; Horta et al., 2019; Vannuccini et al., 2020) e exames bioquímicos como a medição de saliva no cortisol (Dziembowska et al., 2019; Horta et al., 2019; O’donnell et al., 2018; Siart et al., 2017). Nesse sentindo impossibilitou a realização de uma metaanálise, como mencionado na metodologia, mesmo com dados apresentados serem quantitativos. Esse problema de uniformização dos dados é um problema em revisões e na área de saúde mental e um potencial limitação desse estudo, visto que escalas para aferição de saúde mental apresentam diversas divergências, além de variação entre países (Cianconi; Betrò; Janiri; 2020; Rice, 2016).

Essas diferenças podem ser verificadas até mesmo entre mesmas medidas de mensuração, como por exemplo o uso do Questionário de estresse de recuperação para atletas (Recovery-Stress Questionnaire for Athletes - RESTQ), cujo objetivo é avaliar o estresse de recuperação atual do indivíduo (Belz et al., 2018; Codonhato et al., 2018). Entretanto entre países mudanças na aplicação da escala ocorre, Belz et al. (2018) utilizou o questionário na versão alemã reduzida que possui 24 questões, enquanto Codonhato et al. (2018) utilizou versão brasileira de 76 questões, em ambos os estudos foi verificado estresse.

Horta et al., 2019, verificou que que 9 pontuações do Recovery-Stress Questionnaire for Athletes - RESTQ mostraram aumentos significativos durante o treinamento, essa pontuação a subdivida do questionário não foi realizada por Belz et al. (2018) e Codonhato et al. (2018) que analisaram as medidas de pontuação total do questionário e verificaram aumento de estresse mental conforme nível e carga de treinamentos e aproximação de competições.

Tabela 2. Categorização dos artigos quanto aos objetivos, métodos (amostra, caracterização da amostra e dados mensurados) e resultados.

\begin{tabular}{|c|c|c|c|}
\hline Fonte, Data & Objetivo & Método & Resultados \\
\hline Belz et al., 2018 & $\begin{array}{l}\text { Investigar o estresse e a } \\
\text { depressão em atletas e } \\
\text { relação com dor lombar }\end{array}$ & $\begin{array}{l}\text { - } 154 \text { atletas de futebol, badminton, } \\
\text { esqui cross country, atletismo e } \\
\text { ginástica. } \\
\text { - } 51 \% \text { mulheres; idade média = } \\
18,81 \pm 5,05 \text { anos) } \\
\text { - Avaliou-se o estresse de } \\
\text { desempenho, estresse } \\
\text { socioemocional, risco de depressão } \\
\text { e bem-estar psicológico e os dois } \\
\text { parâmetros de dor lombar. }\end{array}$ & $\begin{array}{l}\text { - Atletas apresentaram, um estresse e } \\
\text { variáveis de depressão maiores que } \\
\text { população controle } \\
\text { - } \quad \text { Houve associação entre estresse e } \\
\text { dor nas costas }\end{array}$ \\
\hline
\end{tabular}




\begin{tabular}{|c|c|c|c|}
\hline $\begin{array}{l}\text { Codonhato et al., } \\
2018\end{array}$ & $\begin{array}{l}\text { Estudar a relação entre } \\
\text { resiliência, estresse e lesões } \\
\text { em o contexto do esporte. }\end{array}$ & $\begin{array}{l}\text { - } \text { Oito atletas do sexo feminino } \\
\text { - } \text { (idade de } 20,4 \pm 2,5 \text { ), } \\
\text { - Mensurados medidas do } \\
\text { Questionário de Estresse e } \\
\text { Recuperação, escala de resiliência, } \\
\text { e análise documental de } \\
\text { prontuários de fisioterapia }\end{array}$ & $\begin{array}{l}\text { - } \text { Observou-se alto nível de estresse } \\
\text { nos atletas } \\
\text { - Não houve correlação com a } \\
\text { ocorrência de lesões }\end{array}$ \\
\hline $\begin{array}{l}\text { Dziembowska et } \\
\text { al., } 2019\end{array}$ & $\begin{array}{c}\text { Estudar a resposta } \\
\text { psicofisiológica a } \\
\text { estressor antecipatório } \\
\text { em atletas jovens }\end{array}$ & $\begin{array}{l}\text { - } 55 \text { atletas (25 membros da liga } \\
\text { feminina de vôlei da Polônia com } \\
\text { idade média de } 22 \pm 1,11 \text { anos e } 30 \\
\text { mulheres sedentárias) na fase } \\
\text { folicular de seu ciclo menstrual } \\
\text { - Foram expostos às tarefas } \\
\text { aritméticas mentais como uma } \\
\text { imitação experimental do estressor. } \\
\text { Foram avaliadas por meio de níveis } \\
\text { de ansiedade autorrelatados e } \\
\text { medidas fisiológicas das } \\
\text { concentrações de cortisol salivar e } \\
\text { frequência cardíaca }\end{array}$ & $\begin{array}{l}\text { - A exposição a estressores baseados } \\
\text { em treinamento parece promover a } \\
\text { resposta do cortisol ao estressor } \\
\text { antecipado }\end{array}$ \\
\hline Horta et al., 2019 & $\begin{array}{c}\text { Avaliar a relação entre o } \\
\text { treinamento carga e } \\
\text { desempenho físico, } \\
\text { marcadores bioquímicos, e } \\
\text { estresse psicológico em } \\
\text { jogadores de voleibol de elite }\end{array}$ & 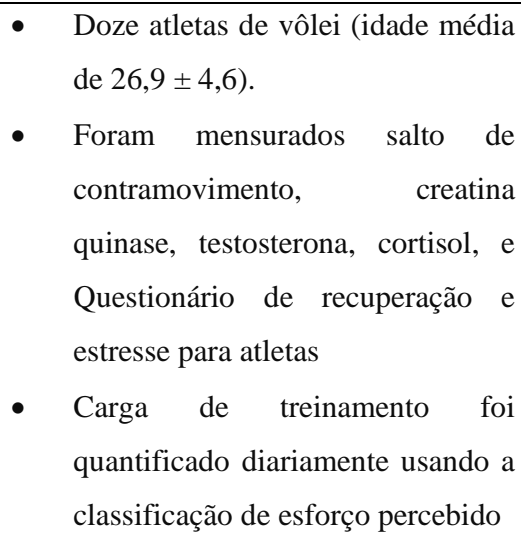 & $\begin{array}{l}\text { - Houve correlação significativa de } \\
\text { magnitude pequena a moderada } \\
\text { entre estresse psicológico e creatina } \\
\text { quinase. } \\
\text { - A carga de treinamento esteve } \\
\text { relacionada com o aumento da } \\
\text { creatina quinase e estresse } \\
\text { psicológico }\end{array}$ \\
\hline $\begin{array}{l}\text { O'donnell et al., } \\
2018\end{array}$ & $\begin{array}{l}\text { Avaliar o cortisol salivar e } \\
\text { marcadores de estresse } \\
\text { perceptivo e índices de sono } \\
\text { durante a competição e } \\
\text { treinamento }\end{array}$ & 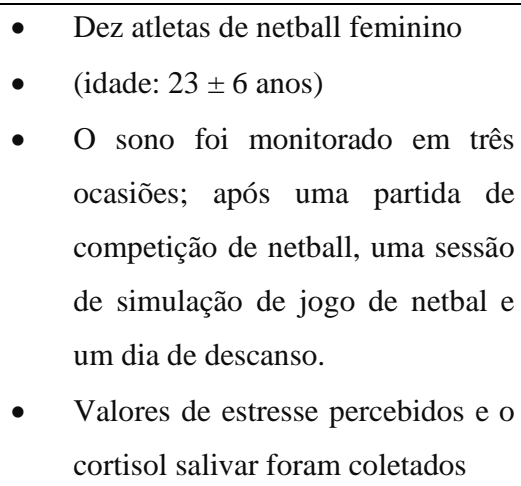 & $\begin{array}{l}\text { - Os níveis de cortisol salivar foram } \\
\text { significativamente mais elevados, e } \\
\text { a quantidade/ qualidade do sono } \\
\text { foram significativamente reduzidas }\end{array}$ \\
\hline Siart et al., 2017 & $\begin{array}{l}\text { Analisar a relação entre } \\
\text { cortisol salivar e níveis de } \\
\text { testosterona e desempenho }\end{array}$ & $\begin{array}{ll}\text { - } & \text { Dezenove atletas de atletismo } \\
\text { - } & \text { (onze homens, oito mulheres idade } \\
& \text { média de } 25,9 \pm 3,9 \text { anos) } \\
\text { - } & \text { Foram analisados cortisol salivar }\end{array}$ & $\begin{array}{l}\text { - O desempenho foi negativamente } \\
\text { correlacionado com o aumento nos } \\
\text { níveis de cortisol. }\end{array}$ \\
\hline
\end{tabular}




\begin{tabular}{|c|c|c|c|}
\hline & & & $\begin{array}{l}\text { - Os níveis de cortisol pré- } \\
\text { competição } \\
\text { significativamente }\end{array}$ \\
\hline $\begin{array}{l}\text { Vannuccini et al., } \\
2020\end{array}$ & $\begin{array}{l}\text { Investigar as características } \\
\text { do ciclo menstrual, estresse } \\
\text { percebido e a qualidade de } \\
\text { vida }\end{array}$ & $\begin{array}{l}\text { - } 112 \text { atletas homens de Futebol, } \\
\text { voleibol, rúgbi, natação, artes } \\
\text { marciais, atletismo, balé e dança } \\
\text { contemporânea e } 103 \text { sedentários } \\
\text { - } \quad \text { (idade média } 19,28 \pm 4,7 \text { anos) } \\
\text { - } \quad \text { Responderam três questionários: } \\
\text { saúde ginecológica, escala de } \\
\text { estresse percebido e qualidade de } \\
\text { vida }\end{array}$ & $\begin{array}{l}\text { - Os atletas tiveram uma melhor } \\
\text { qualidade de vida física, mas maior } \\
\text { nível de estresse percebido e uma } \\
\text { pior qualidade de vida mental, pior } \\
\text { significativamente }\end{array}$ \\
\hline
\end{tabular}

Fonte: Autoria própria.

Outro método utilizado foi a análise de cortisol pela, importante também em detecção de estresse em diferentes áreas da saúde e recorrente investigado em desportos (Siart et al., 2017), o cortisol é um hormônio glicocorticoide liberado pelas glândulas adrenais em resposta ao estresse psicológico e fisiológico Quatros estudos dessa revisão (Dziembowska et al., 2019; Horta et al., 2019; O’donnell et al., 2018; Siart et al., 2017) verificaram o cortisol para medidas de desfechos de estresse, nessas pesquisas o cortisol esteve elevado conforme carga de treinamento ou intensificação de treinamento para competições. Belz et al. (2018) observou que os níveis de cortisol, aumentaram entre 24 horas antes da competição, esse aumento do cortisol em momento tão curto, indica que uma competição é um estressor fisiológico grave.

No estudo de Vannuccini et al. (2019), os autores inferiram que ciclo menstrual, podia interferir negativamente no estresse de mulheres. No entanto nenhuma diferença de gênero foi encontrada em relação ao estresse nos estudos em que população era composta por atletas do sexo masculino e feminino (Belz et al., 2018; Siart et al., 2017). Belz et al. (2019) verificou ainda que houve diferenças em relação à idade, no qual atletas com menos de 18 anos apresentaram menor estresse socioemocional e maior bem-estar psicológico sendo do que atletas mais velhos, nas outras pesquisas não indicaram essa relação idade, é importante ressaltar que a amostra dos outros estudos era composta por jovens adultos (Codonhato et al., 2018; Dziembowska et al., 2019; Horta et al., 2019; O’donnell et al., 2018; Siart et al., 2017; Vannuccini et al., 2020), uma análise em blocos com atletas de elite adoslecentes, jovens e sênior é necessário no futuro.

Uma limitação apontada por Siart et al. (2017) foi a não verificação do sono nos atletas e correlação com estresse mental. Algo que foi verificado no estudo de O'donnell et al. (2018), no qual viu em seus resultados que o ambiente de jogo resultou em níveis pós-jogo de cortisol e sono reduzido quando comparada ao pós-treinamento em atletas de elite

Somente uma pesquisa (Codonhato et al., 2018) analisou a correlação entre estresse mental e ocorrência de lesões, e reparou que não houve correlação entre ao estresse e episódios de lesões, contudo, os atletas por meio do questionário estruturado informaram que acreditavam que o maior estresse podia contribuir para maior temo de recuperação de leões, essa variável também não teve resultado significativo. Essa percepção de que o estresse mental pode influenciar lesões também é relatado pelos atletas de Codonhato et al. (2018).

Em relação ao desempenho em competições Horta et al. (2019) analisou que curto período preparatório foi suficiente para aumentar o estresse mental, mas sem alteração no desempenho físico no campeonato de voleibol de elite. Assim como que relata que período mais longos de treinamentos com menor carga podem apresentar mesma eficácia no desempenho que períodos curtos com maior carga utilizado, corroborando também com afirmado por Codonhato et al. (2018). Siart et al., (2017) verificou 
que os atletas com maior nível de cortisol tiveram pior desempenho no Campeonato Europeu de Atletismo por Equipes no Atletismo, o tipo e carga de treinamento não foi informado

Outro ponto importante foi mostrado por Vannuccini et al. (2020) em que atletas mesmo com qualidade de vida física melhor mostram paramentos piores na qualidade de vida mental. É necessário pesquisa com depressão e qualidade de vida mental em atletas, além do que foi consenso dos artigos incluídos dessa análise sistemática que poucas intervenções e atenção é voltada a saúde mental de atletas de elite. Nesse sentido são necessários ajustes e otimização nas cargas de treinamento, principalmente em treinamentos de preparação próximos a competições, além de uma atenção maior a saúde mental de atletas de elite.

Existem limitações nesses estudo que podem restringir as conclusões que podem ser tiradas nesta revisão, mas também podem indicar os pontos onde pesquisas adicionais são necessárias. Conforme já indicado metodologias diferentes e heterogêneos impediram a aplicação de técnicas meta-analíticas. Ademais, as diferenças entre os esportes incluídos em termos de treinamento, remuneração, pressão da mídia e outras variáveis importantes devem ser consideradas em novas pesquisas. Além disso, o viés dos resultados do avaliador não foi abordado por nenhum dos estudos.

\section{Conclusão}

Nesse estudo de revisão sistemática foi verificado em todos os estudos que atletas possuem nível de estresse mental, e que esse sintoma psicológico parece estar correlacionado com carga de treinamento ou competições. Não houve diferenças entre gêneros em relação ao nível ou percepção de estresse. O estresse mental parece estar correlacionado com carga de treinamento. Estudos com números maiores de atletas categorizados em tipo de esporte, treinamento, faixa etária são necessários.

\section{Referências}

Ahn, E., \& Kang, H. (2018). Introduction to systematic review and meta-analysis. Korean journal of anesthesiology, 71(2), 103.

Åkesdotter, C., Kenttä, G., Eloranta, S., \& Franck, J. (2020). The prevalence of mental health problems in elite athletes. Journal of science and medicine in sport, 23(4), 329-335.

Belz, J., Heidari, J., Levenig, C., Hasenbring, M., Kellmann, M., \& Kleinert, J. (2018). Stress and risk for depression in competitive athletes suffering from back pain-Do age and gender matter?. European journal of sport science, 18(7), 1029-1037.

Cianconi, P., Betrò, S., \& Janiri, L. (2020). The impact of climate change on mental health: a systematic descriptive review. Frontiers in psychiatry, 11.

Codonhato, R., Rubio, V., Oliveira, P. M. P., Resende, C. F., Rosa, B. A. M., Pujals, C., \& Fiorese, L. (2018). Resilience, stress and injuries in the context of the Brazilian elite rhythmic gymnastics. PloS one, 13(12), e0210174.

Delgado-Rodríguez, M., \& Sillero-Arenas, M. (2018). Systematic review and meta-analysis. Medicina Intensiva, 42 (7), $444-453$.

Donato, H., \& Donato, M. (2019). Stages for undertaking a systematic review. Acta medica portuguesa, 32(3), $227-235$.

Dziembowska, I., Wójcik, M., Hołyńska-Iwan, I., Litwic-Kaminska, K., Słomka, A., \& Żekanowska, E. (2019). Female volleyball players are more prone to cortisol anticipatory stress response than sedentary women. Medicina, 55(6), 258.

Foskett, R. L., \& Longstaff, F. (2018). The mental health of elite athletes in the United Kingdom. Journal of science and medicine in sport, 21(8), 765-770.

Gorczynski, P. F., Coyle, M., \& Gibson, K. (2017). Depressive symptoms in high-performance athletes and non-athletes: a comparative meta-analysis. British Journal of Sports Medicine, 51(18), 1348-1354.

Horta, T. A., Bara Filho, M. G., Coimbra, D. R., Miranda, R., \& Werneck, F. Z. (2019). Training load, physical performance, biochemical markers, and psychological stress during a short preparatory period in brazilian elite male volleyball players. The Journal of Strength \& Conditioning Research, 33(12), 33923399 .

Lebrun, F., \& Collins, D. (2017). Is elite sport (really) bad for you? Can we answer the question?. Frontiers in psychology, 8, 324.

Melnyk, B. M., \& Fineout-Overholt, E. (Eds.). (2011). Evidence-based practice in nursing \& healthcare: A guide to best practice. Lippincott Williams \& Wilkins.

Moher, D., Liberati, A., Tetzlaff, J., Altman, D. G., \& Prisma Group. (2009). Preferred reporting items for systematic reviews and meta-analyses: the PRISMA statement. PLoS medicine, 6(7), e1000097. 
Research, Society and Development, v. 10, n. 7, e55710716838, 2021 (CC BY 4.0) | ISSN 2525-3409 | DOI: http://dx.doi.org/10.33448/rsd-v10i7.16838

Moreland, J. J., Coxe, K. A., \& Yang, J. (2018). Collegiate athletes' mental health services utilization: A systematic review of conceptualizations, operationalizations, facilitators, and barriers. Journal of sport and health science, 7(1), 58-69.

O’Donnell, S., Bird, S., Jacobson, G., \& Driller, M. (2018). Sleep and stress hormone responses to training and competition in elite female athletes. European journal of sport science, $18(5), 611-618$

Paiano, M., Jaques, A. E., Nacamura, P. A. B., Salci, M. A., Radovanovic, C. A. T., \& Carreira, L. (2020). Saúde mental dos profissionais de saúde na China durante pandemia do novo coronavírus: revisão integrativa. Revista Brasileira de Enfermagem, 73.

Purcell, R., Rice, S., Butterworth, M., \& Clements, M. (2020). Rates and correlates of mental health symptoms in currently competing elite athletes from the Australian National high-performance sports system. Sports Medicine, 1-12.

Putukian, M. (2016). The psychological response to injury in student athletes: a narrative review with a focus on mental health. British Journal of Sports Medicine, 50(3), 145-148.

Reardon, C. L., Hainline, B., Aron, C. M., Baron, D., Baum, A. L., Bindra, A., \& Engebretsen, L. (2019). Mental health in elite athletes: International Olympic Committee consensus statement (2019). British journal of sports medicine, 53(11), 667-699.

Rice, S. M., Purcell, R., De Silva, S., Mawren, D., McGorry, P. D., \& Parker, A. G. (2016). The mental health of elite athletes: a narrative systematic review. Sports medicine, 46(9), 1333-1353.

Russell, S., Jenkins, D., Rynne, S., Halson, S. L., \& Kelly, V. (2019). What is mental fatigue in elite sport? Perceptions from athletes and staff. European Journal of Sport Science, 19(10), 1367-1376.

Schinke, R. J., Stambulova, N. B., Si, G., \& Moore, Z. (2018). International society of sport psychology position stand: Athletes' mental health, performance, and development. International journal of sport and exercise psychology, 16(6), 622-639.

Siart, B., Nimmerichter, A., Vidotto, C., \& Wallner, B. (2017). Status, stress and performance in track and field athletes during the european games in Baku (Azerbaijan). Scientific reports, 7(1), 1-9.

Stillman, M. A., Glick, I. D., McDuff, D., Reardon, C. L., Hitchcock, M. E., Fitch, V. M., \& Hainline, B. (2019). Psychotherapy for mental health symptoms and disorders in elite athletes: a narrative review. British journal of sports medicine, 53(12), 767-771.

Van Slingerland, K. J., Durand-Bush, N., Bradley, L., Goldfield, G., Archambault, R., Smith, D., \& Kenttä, G. (2019). Canadian Centre for Mental Health and Sport (CCMHS) position statement: Principles of mental health in competitive and high-performance sport. Clinical journal of sport medicine, 29(3), 173-180.

Vannuccini, S., Fondelli, F., Clemenza, S., Galanti, G., \& Petraglia, F. (2020). Dysmenorrhea and heavy menstrual bleeding in elite female athletes: quality of life and perceived stress. Reproductive Sciences, 27(3), 888-894

Vella, S. A., Swann, C., Batterham, M., Boydell, K. M., Eckermann, S., Ferguson, H., \& Deane, F. P. (2021). An intervention for mental health literacy and resilience in organized sports. Medicine and science in sports and exercise, 53(1), 139.

White, K. (2016). Evidence-based practice. Translation of Evidence into Nursing and Health Care. 2nd ed. New York: Springer Publishing Company.

Zhu, X. (2020). Systematic reviews and meta-analysis. Routledge Handbook of Adapted Physical Education. 